\title{
A Hydrodynamic model for a dynamical jammed-to-flowing transition in gravity driven granular media
}

\author{
Lydéric Bocquet $^{(1)}$ Jalal Errami $^{(1)}$ and T. C. Lubensky ${ }^{(2)}$ \\ (1) Laboratoire de Physique (UMR CNRS 5672) \\ ENS-Lyon, 46 allée d'Italie, 69364 Lyon Cedex 07, France \\ (2) Physics Department, University of Pennsylvania, Philadelphia, Pennsylvania 19104, USA
}

(November 21, 2018)

\begin{abstract}
Granular material on an inclined plane will flow like a fluid if the angle $\theta$ the plane makes with the horizontal is large enough. We employ a modification of a hydrodynamic model introduced previously to describe Couette flow experiments to describe chute flow down a plane. In this geometry, our model predicts a jammed-to-flowing transition as $\theta$ is increased even though it does not include solid friction, which might seem necessary to stabilize a state without flow. The transition is driven by coupling between mean and fluctuating velocity. In agreement with experiments and simulations, it predicts flow for layers with a thickness $H$ larger than a critical value $H_{\text {stop }}(\theta)$ and absence of flow for $H<H_{\text {stop }}(\theta)$.
\end{abstract}

In recent years, considerable experimental and theoretical effort has been devoted to the study of flow properties of granular media, yet no fully satisfying phenomenology of granular rheology has emerged [1]. The major difficulty in describing granular flows, particularly in dense media, is that both kinetic phenomena associated with particle motion and more static phenomena like solid friction and force chains are expected to be important. Following early work of Jenkins and Savage and of Haft [2], various kinetic-hydrodynamic threories based on coupled equations for the transport of mementum and kinetic energy have been constructed. They assume instantaneous binary collisions and neglect any solid-like friction between grains. Various authors have proposed including shear independent terms in the stress tensor to capture empirically the effects of intergrain friction [3,4]. Neither of these types of theories incorporates the effects of strongly inhomogenoeus force networks in static and slowly driven dense granular materials [5, 60]

On the basis of experimental results on sheared granular material in Couette geometry, we recently proposed a modified hydrodynamic description [7], whose aim is to include high-density effects in equations describing energy and momentum transport. Specifically, in analogy with the behavior of supercooled liquids close to the glass transition, we assumed an "anomalous" divergence of the viscosity close to random close packing. Even though our approach still does not include enduring contacts and solid friction between grains, it is able to reproduce most of the experimental results in the Couette Geometry.

Granular flow down inclined slopes is of considerable practical importance in geophysical phenomena such as rock and snow avalanches, pyroclastic flows, etc.. Systematic experiments [8] and molecular simulations [10] on a layer of beads on an inclined plane as a function of layer thickness $H$ and angle $\theta$ of the plane relative to horizontal yield a curve $H_{\text {stop }}(\theta)$ separating jammed, stationary behavior at small $H$ from flowing behavior at large $H$. Moreover, precise and systematic measurements of the mean-bead velocity yield a maximum velocity that scales as $H^{3 / 2}$.

In this paper, we analyze chute flow down inclined planes using the frictionless kinetic-hydrodynamic theory developed for the study of Couette flows [7]. Our analysis produces results in agreement with experiment and simulation in this geometry and provides further evidence that our phenomenological kinetic-hydrodynamic theory provides a robust and general description of flowing granular media. In particular, we find an $h_{\text {stop }}$ curve, or equivalently a critical angle separating stationary from flowing behavior. Normally, the existence of such a critical angle $\theta_{c}$ is associated with solid friction: a solid block on an inclined plane is stationary for $\theta<\theta_{c}$ and slides for $\theta>\theta_{c}$. In our analysis, the existence of $\theta_{c}$ is associated with a purely dynamical jammed-to-flowing transition in which solid friction is totally absent. The origin of this transition is the coupling between velocity fluctuations and mean flow - an important feature of our previous study.

Geometry of the problem. We consider the geometry of the experiments in Refs. [8]: a layer of granular material, composed of spheres of diameter $d$, of thickness $H$ flows on an inclined plane making an angle $\theta$ with the horizontal. We choose a coordinate system with the $x$-axis parallel to the plane, the $z$-axis perpendicular to it, and origin at the bottom of the flowing layer. In a stationary state, conservation of momentum in directions parallel and perpendicular to the plane leads to the equations: $\partial_{z} \sigma_{x z}=\rho(z) g \sin \theta$ and $\partial_{z} \sigma_{z z}=\rho(z) g \cos \theta$, where $\rho$ is the mass density and $\sigma_{z z}$ and $\sigma_{x z}$ are, respectively, the 
diagonal and off diagonal components of the stress tensor. Combining these two model-independent equations yields the simple relation: $\sigma_{x z}=\tan \theta \sigma_{z z}+C$, where $C$ is a constant independent of $z$. In our hydrodynamic model, the off diagonal component of the stress tensor is viscous like: $\sigma_{x z}=\eta(\rho, T) \dot{\gamma}$, where $\dot{\gamma}=d V_{x} / d z$ is the shear rate, $V_{x}$ the mean velocity, and $\eta$ is the viscosity, a function of the density and granular temperature field $T$. As usual the latter is defined in terms of the RMS part of the velocity field $T(z)=\frac{1}{3} m\left\langle v^{2}(z)\right\rangle$. The temperature profile is determined by the balance between viscous heating, heat flow, and dissipation through inelastic collisions [7]: $\frac{\partial}{\partial z} \lambda(\rho, T) \frac{\partial}{\partial z} T+\sigma_{x z} \dot{\gamma}-\epsilon(\rho, T) T=0$, where $\lambda(\rho, T)$ is the thermal conductivity and $\epsilon(\rho, T)$ is the rate of kinetic energy loss. These hydrodynamic equations are closed by the equation of state $P=f(\rho) T$. In contrast to classical thermalized fluids, there are strong temperature and density variations over the system, and the density and temperature dependence of transport coefficients play an important role in determining flow properties. These dependences are usually obtained using kinetic theory [2] with the Enskog approximation for the collision kernel, which accounts for excluded volume but neglects any correlation between collisions. By construction this theory yields transport coefficients that are roughly proportional to the collision frequency, i.e., to the pair-correlation function at contact, which in the high-density limit is expected to diverge like $\left(\rho_{c}-\rho\right)^{-1}$ close to the maximum density, $\rho_{c}$, allowed by excluded volume effects [11]. The assumption of negligible correlation between collisions should fail at high density, and in our modified description of Ref. [7], we proposed replacing the Enskog expression for the viscosity with one with a specific "anomalous" algebraic divergence, $\eta \sim\left(\rho_{c}-\rho\right)^{-\beta}$ [13] close to $\rho_{c}$, in full analogy with the behavior of supercooled liquids close to the glass transition [12]. This picture is supported by the experimental observation in sheared granular material of a specific scaling law relating the local shear rate $\dot{\gamma}$ to the granular temperature $T$ [7.,15].

In the Couette geomety, the density is high throughout the cell. In contrast, in the current chute geometry, small densities could in principle be reached at the top surface, and we need to include the low-density limit in our expressions for transport coefficients. Since our aim is to provide a sketch of the jammed-to-flowing, we shall not use the full Enskog expression for transport coefficient in the small and intermediate density regions, as is done by various authors [2]. Instead, we will use simple interpolated expressions that are consistent with both the high-, intermediate-, and small-density limit. Our specific choices are $\lambda(\rho, T)=\left[\lambda_{0} /\left(m^{1 / 2} d^{2}\right)\right](1-$ $\left.\rho / \rho_{c}\right)^{-1} T^{1 / 2}, \epsilon(\rho, T)=\left[\epsilon_{0} /\left(m^{1 / 2} d\right)\right] \rho\left(1-\rho / \rho_{c}\right)^{-1} T^{1 / 2}$, $\eta(\rho, T)=\eta_{0}\left[m^{1 / 2} / d^{2}\right] g(\rho) T^{1 / 2}$, where $m$ the mass of one particle, $d$ is its diameter, and $\lambda_{0}, \epsilon_{0}$ and $\eta_{0}$ are dimensionless constants. The function $g(\rho)$ is chosen so as to interpolate between the very low-density limit, where the viscosity goes to a constant, to its highdensity "anomalously" diverging behavior close to $\rho_{c}$, passing through its Enskog behavior for intermediate (but already high) densities. A sensible choice is simply $g(\rho)=\left(\alpha(\rho)^{\beta-1}+\alpha_{0}^{\beta-1}\right) \alpha(\rho)^{-\beta} /\left(1+\alpha_{0}^{\beta-1}\right)$, where $\alpha(\rho)=1-\rho / \rho_{c}$ and $\beta$ is the anomalous exponent discussed above. The parameter $\alpha_{0}$ fixes the density where the crossover to the anomalous regime occurs. The equation of state is written as $P / T=\rho /\left(1-\rho / \rho_{c}\right)$, which does indeed reduce to the correct limits in the low- and high-density limits [11]. We emphasize that our results are not very sensitive to the specifics forms for $g$ and the equation of state. Our expressions were chosen to minimize the number of parameters in the model.

The previous equations can be made dimensionless using a reduced velocity $v=V / \sqrt{(g d)}$, temperature $t=$ $T /(m g d)$, stress $\sigma=\sigma_{x z} /\left(\rho_{c} g d\right)$, pressure $p=P /\left(\rho_{c} g d\right)$, and distance $\tilde{z}=z / d$ (we shall omit the tilde over $\tilde{z}$ in the following). Using the previously defined expressions for the transport coefficients, we can now rewrite the coupled equations for stress and temperature as:

$$
\begin{gathered}
\frac{\partial}{\partial z} p=-\cos \theta \frac{t}{p+t} \\
\partial_{z}\left(\left(\frac{p+t}{t^{1 / 2}}\right) \partial_{z} t\right)+a \frac{\sigma^{2}}{g\left(\frac{p}{p+t}\right) t^{1 / 2}}-b p t^{1 / 2}=0
\end{gathered}
$$

where $p /(p+t)=\rho / \rho_{c}$ is the reduced density, $a=$ $\left(\rho_{c} d^{3}\right)^{2} /\left(\lambda_{0} \eta_{0}\right)$ and $b=\epsilon_{0} \rho_{c} d^{3} / \lambda_{0}$ are dimensionless constants, $\sigma$ is related to $p$ according to $\sigma=\tan \theta p+c$, with $c$ an as yet unspecified constant. The parameters appearing in Eq. (1) are strongly constrained by the complementary experiments performed in the Couette cell [7, 15]. In this case, the velocity and temperature profiles are localized close to the moving boundary over a depth of a few particles diameter. This behavior is predicted by the hydrodynamic model with a characteristic decay lentgh for the RMS velocity given by $\delta / d=(2 / b)^{1 / 2}[$ []. Experimentally $\delta$ is of the order of 4-5 particle diameters, yielding $b \simeq 0.1$ (we shall use in the following $b=0.111$ ). The exponent $\beta$ was determined experimentally in Ref. [7] to be 1.75 , while experiments over a larger range of data by Mueth yielded $\beta=1.5$. The parameter $\alpha_{0}$ is chosen such that the cross over to the anomalous scaling occurs roughly $10 \%$ below random close packing. In pratice $\alpha_{0}=0.05$ is adequate. Eventually, $a$ is expected to be of order unity.

The hydrodynamic equations (11) have to be supplemented by boundary conditions for the velocity and temperature fields at the bottom and top surfaces. As to the conditions on the granular temperature, we showed in our previous study of the Couette cell that a vanishing "heat flux" condition at the boundaries yielded results in agreement with the experimental ones. In the present study, we shall thus assume $\partial_{z} T=0$ at the boundaries. In the recent simulations of Silbert et al. [10], the temperature 
profile has a vanishing derivative close to the bottom and top boundaries. The previous assumption thus amounts to slightly shifting the hydrodynamic boundaries of the system, a behavior which is encountered in classical fluids [14]. The boundary condition for the mean velocity field are a priori more obvious: at the wall surface, the velocity is assumed to vanish, while at the top surface a stress-free boundary condition will be assumed, $\sigma=0$, yielding $\partial_{z} v=0$ at $z=H$. Since, as mentioned above, the effective hydrodynamic boundary $z=H$ is located slightly inside the material, the pressure is not expected to vanish there, and it can be written as $p_{0}=z_{0} \cos \theta$ (in reduced units) with $z_{0}$ a molecular distance of the order of the diameter (unity in reduced units). To be fully consistent with the stress free boundary for the velocity, we thus write $\sigma=\tan \theta\left(p-p_{0}\right)$ (i.e., fix the constant $\left.c=-\tan \theta p_{0}\right)$. As emphasized above, this is a molecular effect that disappears in the large- $H$ limit. Moreover this choice does not affect the existence of the jammedto-flowing transition to be discussed now.

The previous system of equations (1) has been solved using a standard Runge Kutta algorithm, using a shooting-like procedure: for a given temperature $T(H)$ at the top boundary, the derivative of $T$ at the bottom, $\partial_{z} T(0)$ is computed. The solutions we seek thus correspond to zeros in the $\partial_{z} T(0)$ versus $T(H)$ curve. A transition between two kinds of behaviour is then found: for a set $\{H, \theta\}$ such that $H$ is below a critical $H_{\text {stop }}(\theta)$ curve, only the $T=0$ solution exists. This corresponds to a "jammed" state, which stays at rest. Above this critical value, nonzero solutions for the temperature, which correspond to a flowing regime do exist. In Fig. 1, we plot the boundary between the two regimes, denoted $H_{\text {stop }}(\theta)$ as in Ref. [8]. This curve is in qualitative agreement with the experimental results of Refs 8 .

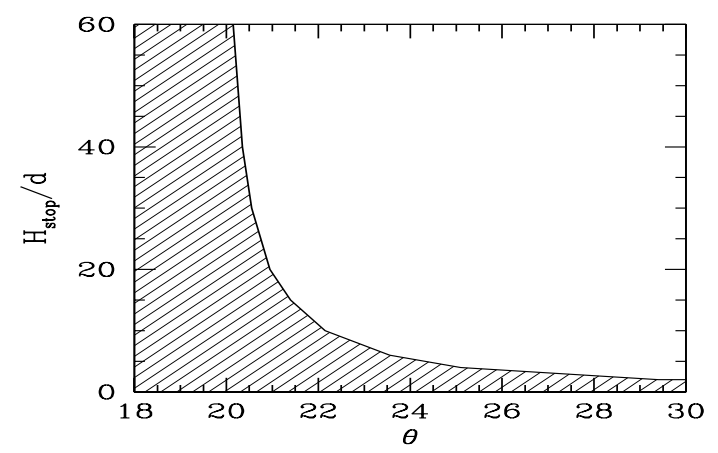

FIG. 1. Phase diagram for the jammed-to-flowing transition in a gravity driven granular material as obtained from the resolution of the hydrodynamic equations (11). The parameters used are $a=1.3, b=0.111, \alpha_{0}=0.05, z_{0}=0.5$.

This shows that the hydrodynamic model is able to generate a finite critical angle below which no flow occurs. Within the model, the origin of this behavior is simply the balance between the "viscous heating", which generates fluctuations, and dissipation through collisions, which tends to inhibit the flow. The transition is thus purely dynamical: a jammed state occurs for low angles/thicknesses because fluctuations are insufficient to allow flow.

Above $H_{\text {stop }}(\theta)$, more than one solution can be found for some sets of parameters $\{H, \theta\}$. However a linear stability analysis of the hydrodynamic equation for the temperature shows that only a single nonzero solution is dynamically stable. On the other hand the $T=0$ solution is always linearly stable but becomes unstable against larger "kicks" in the temperature. It is important to note that there is a solution to the hydrodynamic equations for any set of $H$ and $\theta$ above the boundary $H_{\text {stop }}(\theta)$, as is found experimentally. This is in contrast to other approaches where for a given $\theta$ a single flowing thickness $H$ is selected [3]. In the flowing regime, we obtain temperature and density profiles, which are plotted in Fig. 2 for $H=40$.

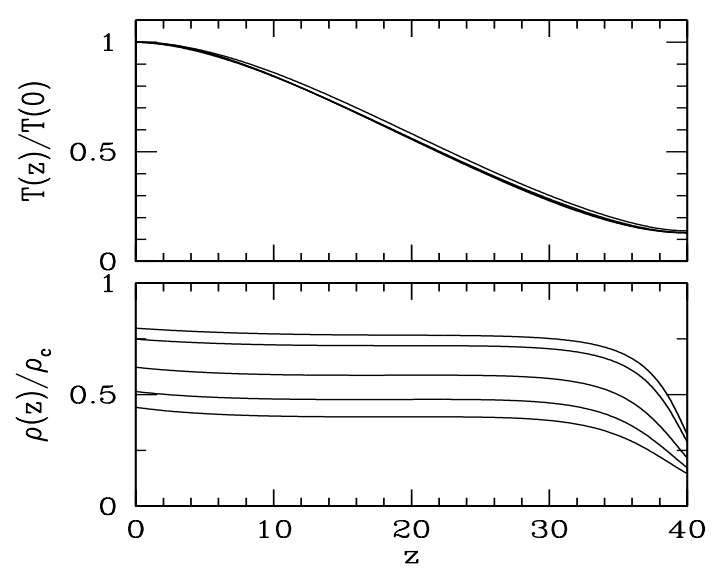

FIG. 2. Top : Normalized temperature profiles for $H=40$, $\theta=20.55,20.90,21.50,22.50$. Bottom : Normalized density profiles for $H=40, \theta=20.55,20.90,22.50,24.50,26.50$ from top to bottom. $\left(a=1.3, b=0.111, \alpha_{0}=0.05, z_{0}=0.5\right)$

Both results for the density and temperature are in qualitative agreement with the simulations of Refs. [10]. In particular the density profile is found to be almost constant in the middle of the sample, with a large drop at the top surface, as found numerically. In the simulations however, the plateau in the density is flatter than ours.

Velocity profiles are obtained by integrating the shear rate $\dot{\gamma}=d v_{x} / d z$, which in our model is linearly related to the non diagonal stress $\sigma_{x z}(z)=\eta(\rho(z), T(z)) \dot{\gamma}(z)$. We find that the $z$ dependence of the profiles is in very good agreement with the predictions of a simple Bagnold scaling $\sigma=A_{\mathrm{Bag}} \dot{\gamma}^{2}, v(z) / v_{\max }=1-(1-z / H)^{3 / 2}$. Within the Bagnold assumption, $v_{\max }=(2 / 3) A_{\mathrm{Bag}} \sqrt{\sin \theta} H^{3 / 2}$. This agreement simply indicates that once the system flows, Bagnold-like scaling is a good approximation to the flow properties, even though it cannot predict a jammed- 
to-flowing transition. The same agreement with Bagnold scaling was observed in the simulations of Ref. 10. The $H^{3 / 2}$ dependence of the maximum velocity $v_{\max }$ is also recovered in our model in agreement with experiments and simulations. Our results are also consistent with a $\sqrt{\sin \theta}$ dependence of $v_{\max }$ with $A_{\mathrm{Bag}} \approx 0.2$. This is in fair agreement with the simulations of Silbert et al. [10], who find a slight dependence of $A_{\mathrm{Bag}}$ on $\theta$. On the other hand, the experiments of Pouliquen exhibit a stronger dependence on $\theta$, with $v_{\max }$ scaling like $\left[H_{\text {stop }}(\theta)\right]^{-1}$

Discussion. Reference [7] introduced a modified kinetic-hydrodynamic theory for granular flow that predicted temperature and velocity profiles in dense systems in Couette geometry in agreement with experiments. In this paper, we calculate the corresponding profiles for flow down an inclined plane using a generalization of this theory to include gravity and low- as well as intermediateand high-density regimes. Our results agree with those obtained in experiments [8] and simulations [10]. Thus, the kinetic-hhydrodynamic theory of granular flow provides a good description of flow in two qualitatively different geometries. Though it is premature to generalize from two to all geometries, it would, nevertheless, appear that this description might serve as an all purpose phenomenological theory for granular flow. In the case of flow down a plane, it is able, contrary to intuition, to predict a jammed-to-flowing transition and a critical angle below which no flow takes place, even though it does not include solid friction between grains, which might be viewed as necessary to stabilize a state without flow.

Our results raise a number of basic questions about the mechanisms underlying granular flows. Two important features of granular materials are (i) the possibility of solid friction between grains and (ii) the absence of velocity fluctuations (i.e., temperature) when the system is at rest. Under flow, the effects of these features become intertwined, making it difficult to provide first principles modeling of the rheology of granular materials. The fact that our frictionless hydrodynamic model produces successful predictions for two different geometries suggests that solid friction is not necessarily a dominant feature (though it may not be negligible). On the other hand, our theory does highlight the importance of coupling between velocity fluctuations and mean flow. Intuitively, one might expect this to be a generic feature of rheology of " $T=0$ " glassy systems: in order to flow, a system must create its own fluctuations, which in turn couple to flow. It is interesting to note that various descriptions based on similar ideas have recently emerged in attempts to provide a phenomenological description of the rheology of complex systems such as gels, paste 17, and foam [18]. The hydrodynamic model successfully captures coupling between mean and RMS velocity fields, and it should serve as a basis for developing improved models that could for example include contact forces. At high density, enduring contacts occur, but they must not be confused with the existence of solid friction between grains. Indeed, enduring contacts do not invalidate a hydrodynamic description; they can be taken into account in transport equations (as done for example in simple liquids with continuous interactions 16 ). In this case, only the $T^{1 / 2}$ scaling of transport coefficients, which stems from the assumption of binary collisions, will be modified, and a more complex temperature dependence of transport coefficients is expected.

L.B. acknowledges fruitful discussions with $\mathrm{O}$. Pouliquen, Y. Forterre and G. Debregeas. T.C.L. acknowledges the support of the National Science Foundation under Grant DMR00-96532 and of the E.N.S. de Lyon, where this work was initiated.

[1] E. Clement, Curr. Opinion in Coll. and Interface Sci. 4, 294 (1999).

[2] J.T. Jenkins and S.B. Savage, J. Fluid Mech. 130186 (1983); P.K. Haft, J. Fluid Mech. 134401 (1983)

[3] P.C. Johnson, P. Nott and R. Jackson, J. Fluid. Mech. 210501 (1991); K.G. Anderson and R. Jackson, J. Fluid Mech. 241, 145 (1992).

[4] I.S. Aronson and L.S. Tsimring, Phys. Rev. E 64020301 (2001).

[5] H.M. Jaeger, S.R. Nagel and R.P. Behringer, Rev. Mod. Phys. 68, 1259 (1996).

[6] P. Mills, D. Loggia, M. Tixier, Europhys. Lett. 45, 733 (1999); G. Debregeas and C. Josserand, Europhys. Lett. 52, 137 (2000).

[7] W. Losert, L. Bocquet, T.C. Lubensky and J.P. Gollub, Phys. Rev. Lett. 851428 (2000); L. Bocquet et al., Phys. Rev. E, in press (2001).

[8] O. Pouliquen, Physics of Fluids, 11542 (1999); A. Daerr and S. Douady, Nature 399241 (1999); E. Azanza, F. Chevoir, P. Moucheron, J. Fluid. Mech. 400199 (1999).

[9] P.A. Lemieux and D. Durian, Phys. Rev. Lett. 85, 4179 (2001)

[10] D. Ertaz et al. Europhys. Lett. 56, 214 (2001); L. Silbert et al. condmat/0105071

[11] R.J. Speedy, J. Chem. Phys. 110, 4559 (1999).

[12] W. Goetze and L. Sjoegren, Rep. Prog. Phys. 55, 241 (1992).

[13] Note that this anomalous behavior is not expected to occur fot the the energy transport coefficients $(\lambda$ and $\epsilon)$. See Ref. [7] for a full discussion concerning this point.

[14] L. Bocquet and J.L. Barrat, Phys. Rev. E 49, 3079 (1994).

[15] D. Müth, condmat/0103557.

[16] J.P. Hansen and I.R. Mc Donald, Theory of Simple Liquids, 2nd ed. (Academic Press, London, 1986).

[17] C. Derec, A. Ajdari, F. Lequeux, Eur. Phys. Jour. E 4, 355 (2001).

[18] G. Debrégeas, H. Tabuteau, J.M. di Meglio, Phys. Rev. Lett. 87, 8305 (2001). 